\title{
THE FIBRED PRODUCT NEAR-RINGS AND NEAR-RING MODULES FOR CERTAIN CATEGORIES
}

\author{
by JAMES R. CLAY* \\ (Received 15th September 1978)
}

\section{Near-rings from group objects}

In some categories, there are structures that look very much like groups, and they usually are. These structures are called group-objects and were first studied by Eckmann and Hilton (1). If our category $\mathscr{C}$ has an object $T$ such that $\operatorname{hom}(X, T)=$ $\left\{t_{x}\right\}$, a singleton, for each object $X \in \mathrm{Ob} \mathscr{C}, T$ is called a terminal object. Our category $\mathscr{C}$ must have products; i.e. for $A_{1}, \ldots, A_{n} \in \mathrm{Ob} \mathscr{C}$, there is an object $A_{1} \times \cdots \times A_{n} \in$ Ob $\mathscr{C}$ and morphisms $p_{i}: A_{1} \times \cdots \times A_{n} \rightarrow A_{i}$ so that if $f_{i}: X \rightarrow A_{i}, i=1,2, \ldots, n$, are morphisms of $\mathscr{C}$, then there is a unique morphism $\left[f_{1}, \ldots, f_{n}\right]: X \rightarrow A_{1} \times \cdots \times A_{n}$ such that $p_{i} \circ\left[f_{1}, \ldots, f_{n}\right]=f_{i}$ for $i=1,2, \ldots, n$.

In the case where $A_{1}=A_{2}=A$, and $f_{1}=f_{2}=1_{A}$, we call $\Delta=\left[1_{A}, 1_{A}\right]$ the diagonal map.

If our category $\mathscr{C}$ has a terminal object $T$ and products, there is a chance that it may have group-objects. By a group-object, we mean a quadruple $(G, \pi, \mu, \alpha)$ where $G \in \mathrm{Ob} \mathscr{C}, \pi \in \operatorname{hom}(G \times G, G)$ is a morphism analogous to the "binary operation," $\mu \in \operatorname{hom}(T, G)$ suggests the "identity," and $\alpha \in \operatorname{hom}(G, G)$ abstracts "inverses." To make a successful analogy, the following diagrams must be commutative.

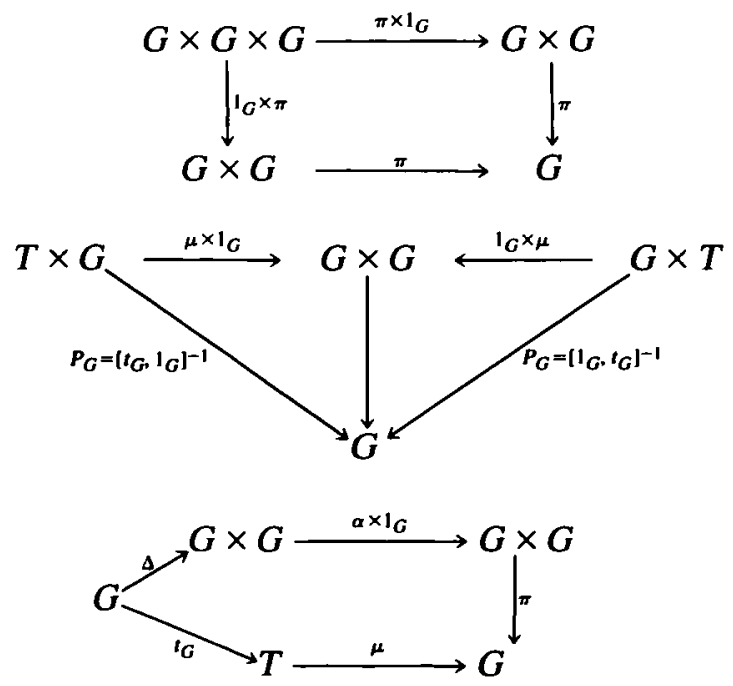

(associativity)

(identity)

(inverses)

*The research was supported by a Senior Scientist Award administered by the Humboldt Foundation of West Germany. 
In the above diagrams, $f \times g: A \times B \rightarrow A^{\prime} \times B^{\prime}$ means $f \times g=\left[f \circ p_{A}, f \circ p_{B}\right]$.

If $G$ is a group object, then $\operatorname{hom}(X, G)$ is a genuine group, with the correct + . The best way to see this + is from the following diagram.

$$
\begin{gathered}
\operatorname{hom}(X, G) \times \operatorname{hom}(X, G) \cong \operatorname{hom}(X, G \times G) \stackrel{\pi^{*}}{\longrightarrow} \operatorname{hom}(X, G) \\
(f, g) \longrightarrow[f, g] \longrightarrow \sigma[f, g]
\end{gathered}
$$

Define $f+g=\pi \circ[f, g], 0=\mu \circ t_{X}$, and $-f=\alpha \circ f$. Then (hom $(X, A),+$ ) is a group.

Now suppose $f, g \in \operatorname{hom}(X, G)$ and $h \in \operatorname{hom}(Y, X)$. Then $(f+g) \circ h=$ $\pi \circ[f, g] \circ h=\pi \circ[f \circ h, g \circ h]=(f \circ h)+(g \circ h)$. (See the next commutative diagram.) So $\circ$ is right distributive over + and therefore (End $G,+, \circ$ ) is a near-ring, and each (hom $(X, G),+$ ) is a left (End $G$ ) group. Now $\operatorname{hom}(-, G)$ is a functor from $\mathscr{C}$ to the category of left (End $G$ ) groups.

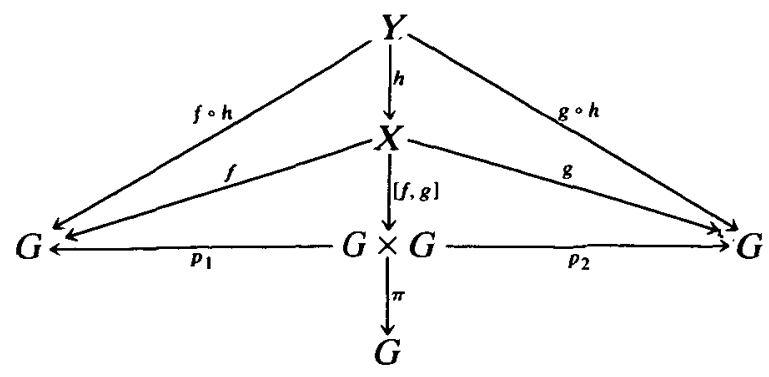

In the category of groups, the group objects are the abelian groups. In the category of sets $\mathscr{P}$, the category of groups $\mathscr{G}$, and the category of abelian groups $\mathscr{A}$, it turns out that $f+g=\pi \circ[f, g]$ is exactly pointwise addition of functions. In this paper, we shall see examples where this is not the case, so our + is a natural and real generalization. Since the pointwise addition of two endomorphisms is not necessarily an endomorphism, we shall see that this definition of + is exactly what is needed.

\section{The fibred product near-ring}

Let $A$ be a fixed object in a concrete category $\mathscr{C}$. From $A$ and $\mathscr{C}$ one constructs a new category $\mathscr{C}(A)$ whose objects are pairs $(X, \eta)$ where $X$ is an object of $\mathscr{C}$ and $\eta: X \rightarrow A$ is an epimorphism. A morphism $f \in \operatorname{hom}((X, \eta),(Y, \epsilon))$ is morphism from $\mathscr{C}$ with the additional property that $\epsilon \circ f=\eta$. That is, we want the following diagram to be commutative.

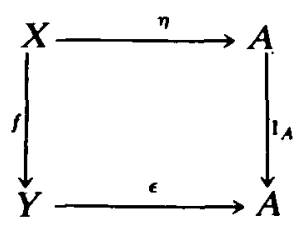

It is direct to show that $\mathscr{C}(A)$ is a category and that $\left(A, 1_{A}\right)$ is a terminal object. We shall now see that products exists in $\mathscr{C}(A)$; this product is called the fibred product. Let $\left(X_{1}, \eta_{1}\right),\left(X_{2}, \eta_{2}\right)$ be two objects of $\mathscr{C}(A)$. (We identify each object $X \in \mathscr{C}$ with its 
set.) The product of $\left(X_{1}, \eta_{1}\right)$ and $\left(X_{2}, \eta_{2}\right)$ is $\left(\left(X_{1} \times{ }_{A} X_{2}, \eta\right), p_{1}, p_{2}\right)$ where

$$
\begin{gathered}
X_{1} \times{ }_{A} X_{2}=\left\{\left(x_{1}, x_{2}\right) \mid \eta_{1}\left(x_{1}\right)=\eta_{2}\left(x_{2}\right)\right\}, \\
\eta: X_{1} \times{ }_{A} X_{2} \rightarrow A
\end{gathered}
$$

is defined by $\eta\left(x_{1}, x_{2}\right)=\eta_{1}\left(x_{1}\right)\left(=\eta_{2}\left(x_{2}\right)\right)$,

and

$$
p_{i}: X_{1} \times{ }_{A} X_{2} \rightarrow X_{i}
$$

is defined by $p_{i}\left(x_{1}, x_{2}\right)=x_{i}$.

It is direct to see that this is a product for $\mathscr{C}(A)$.

Note that hom $\left((X, \eta),\left(A, 1_{A}\right)\right)=\{\eta\}$, so $t_{(X, \eta)}=\eta$. Also $[f, g]: Y \rightarrow X_{1} \times{ }_{A} X_{2}$ is given by $[f, g](y)=(f(y), g(y))$, and $\Delta: X \rightarrow X \times{ }_{A} X$ is given by $\Delta(x)=(x, x)$.

If $((G, \gamma), \pi, \mu, \alpha)$ is a group object of $\mathscr{C}(A)$, the endomorphism near-ring (End $((G, \gamma)),+, \circ)$ is called a fibred product near-ring.

The remainder of the paper is concerned with determining the structure of (1) group objects $(G, \gamma)$, (2) the fibred product near-rings $\operatorname{End}((G, \gamma))$, and (3) End $((G, g))$ - groups $\operatorname{hom}((X, \eta),(G, \gamma))$. We do this first when $\mathscr{C}$ is $\mathscr{S}$, the category of sets, and then when $\mathscr{C}$ is $\mathscr{G}$, the category of groups.

\section{The fibred product near-rings for $\mathscr{S}$, the category of sets}

Fix a set $A$. We'll first determine the group objects of $\mathscr{P}(A)$.

Suppose $((G, \gamma), \pi, \mu, \alpha)$ is a group object of $\mathscr{S}(A)$. First look at $\mu$ for a moment. Now $\mu \in \operatorname{hom}\left(\left(A, 1_{A}\right),(G, \gamma)\right)$ and so $\gamma \circ \mu=1_{A}$. Thus $\mu(a) \in \gamma^{-1}(a)$ for each $a \in A$. That is, $\mu$ selects exactly one element from each of the family $\left\{\gamma^{-1}(a) \mid a \in A\right\}$. We shall return to $\mu$, but let us now turn our attention to $\pi$.

Since $\pi \in \operatorname{hom}\left(\left(G \times{ }_{A} G, \bar{\gamma}\right),(G, \gamma)\right)$, we have $\gamma \circ \pi=\bar{\gamma}$. For $\left(x_{1}, x_{2}\right) \in G \times{ }_{A} G$, one has that $\gamma\left(x_{1}\right)=\gamma\left(x_{2}\right)$. But this means that $\left(x_{1}, x_{2}\right) \in G \times{ }_{A} G$ if and only if $x_{1}, x_{2} \in$ $\gamma^{-1}(a)$ for $a=\gamma\left(x_{1}\right)=\gamma\left(x_{2}\right)$. This means that

$$
G \times{ }_{A} G=\bigcup_{a \in A}\left[\gamma^{-1}(a) \times \gamma^{-1}(a)\right] .
$$

Now $\gamma\left(x_{1}\right)=\bar{\gamma}\left(x_{1}, x_{2}\right)=\gamma\left(\pi\left(x_{1}, x_{2}\right)\right)$ simply means that if $x_{1}, x_{2} \in \gamma^{-1}(a)$, then so is $\pi\left(x_{1}, x_{2}\right) \in \gamma^{-1}(a)$. Hence $\pi$ defines a family of binary operations $\left\{\pi_{a} \mid a \in A\right\}$ where $\pi_{a}: \gamma^{-1}(a) \times \gamma^{-1}(a) \rightarrow \gamma^{-1}(a)$. We have so far a family of systems $\left\{\left(\gamma^{-1}(a), \pi_{a}, \mu(a)\right) \mid a \in\right.$ $A\}$ where $\pi_{a}$ is a binary operation on $\gamma^{-1}(a)$ and $\mu(a) \in \gamma^{-1}(a)$. Let us now look at the "identity diagram" for our group object.

We have

$$
x=p_{G}(a, x)=\pi \circ \mu \times 1_{G}(a, x)=\pi(\mu(a), x)=\pi_{a}(\mu(a), x)
$$

and

$$
x=p_{G}(x, a)=\pi \circ 1_{G} \times \mu(x, a)=\pi(x, \mu(a))=\pi_{a}(x, \mu(a)) .
$$

Hence $\mu(a)$ is an identity for $\left(\gamma^{-1}(a), \pi_{a}\right)$.

Looking at the "associative diagram" for $\pi$, we see that we must have $\pi \circ \pi \times 1_{G}=$ $\pi \circ 1_{G} \times \pi$. Take $\left(x_{1}, x_{2}, x_{3}\right) \in G \times{ }_{A} G \times{ }_{A} G$. Then $\gamma\left(x_{1}\right)=\gamma\left(x_{2}\right)=\gamma\left(x_{3}\right)=a$ and so $x_{1}, x_{2}, x_{3} \in \gamma^{-1}(a)$. Hence $\pi \circ \pi \times 1_{G}\left(x_{1}, x_{2}, x_{3}\right)=\pi\left(\pi\left(x_{1}, x_{2}\right), x_{3}\right)=\pi_{a}\left(\pi_{a}\left(x_{1}, x_{2}\right), x_{3}\right)$ 
and

$$
\pi \circ 1_{G} \times \pi\left(x_{1}, x_{2}, x_{3}\right)=\pi_{a}\left(x_{1}, \pi_{a}\left(x_{2}, x_{3}\right)\right) .
$$

We have just seen that each $\pi_{a}$ is associative.

Turning our attention now to the "inverse diagram" for $\alpha$, we must have $\pi \circ \alpha \times$ $1_{G} \circ \Delta=\mu \circ t_{(G, \gamma)}$. Here $t_{(G, \gamma)}=\gamma$. So

$$
\pi \circ \alpha \times 1_{G} \circ \Delta(x)=\pi \circ \alpha \times 1_{G}(x, x)=\pi(\alpha(x), x)
$$

and

$$
\mu \circ t_{(G, \gamma)}(x)=\mu(\gamma(x))=\mu(a)
$$

where $x \in \gamma^{-1}(a)$. So we see that $x \in \gamma^{-1}(a)$ implies $\alpha(x) \in \gamma^{-1}(a)$ and so

$$
\mu(a)=\pi(\alpha(x), x)=\pi_{a}(\alpha(x), x) .
$$

Since $\mu(a)$ is the identity of $\left(\gamma^{-1}(a), \pi_{a}\right)$, we have that for each $a \in A$, $\left(\gamma^{-1}(a), \pi_{a}, \mu(a), \alpha \mid \gamma^{-1}(a)\right)$ is a group with the restriction $\alpha$ to $\gamma^{-1}(a), \alpha \mid \gamma^{-1}(a)$, giving inverses with respect to $\pi_{a}$ and $\mu(a)$.

This gives us half of the following

Theorem 1. The group objects $((G, \gamma), \pi, \mu, \alpha)$ of $\mathscr{P}(A)$ are essentially any family $\left\{\left(\gamma^{-1}(a), \pi_{a}, \mu(a), \alpha_{a}\right) \mid a \in A\right\}$ of groups where $G=\cup_{a \in A} \gamma^{-1}(a), \mu(a) \in \gamma^{-1}(a), \alpha_{a}=$ $\alpha \mid \gamma^{-1}(a) ;$ and $\pi_{a}=\pi \mid \gamma^{-1}(a) \times \gamma^{-1}(a)$.

Proof. Let $(G, \gamma)$ be an object of $\mathscr{S}(A)$. So $\gamma: G \rightarrow A$ is a surjection, and if $a \in A, \gamma^{-1}(a) \neq \emptyset$. Start with the family $\left\{\gamma^{-1}(a) \mid a \in A\right\}$, a partition on $G$. For each $a \in A$, let $\pi_{a}$ be a binary operation on $\gamma^{-1}(a)$ so that $\left(\gamma^{-1}(a), \pi_{a}\right)$ is a group, and let $\pi=\cup_{a \in A} \pi_{a}$. Then $\pi(x, y)=z$ means that $\pi_{a}(x, y)=z$ for some $a \in A$ where $x, y, z \in$ $\gamma^{-1}(a)$. Hence $\pi: G \times{ }_{A} G \rightarrow G$ and $\gamma \circ \pi=\bar{\gamma}$, giving $\pi \in \operatorname{hom}\left(\left(G \times{ }_{A} G, \bar{\gamma}\right),(G, \gamma)\right)$. It is direct to see that $\pi \circ \pi \times 1_{G}=\pi \circ 1_{G} \times \pi$, so the "associative diagram" is commutative.

Define $\mu: A \rightarrow G$ by setting $\mu(a)$ equal to the identity element of the group $\left(\gamma^{-1}(a), \pi_{a}\right)$. Since $\mu(a) \in \gamma^{-1}(a)$, it follows that $\mu \in \operatorname{hom}\left(\left(A, 1_{A}\right),(G, \gamma)\right)$. Now $\pi \circ \mu \times$ $1_{G}(a, g)=\pi(\mu(a), g)=\pi_{a}(\mu(a), g)=g=p_{G}(a, g)$, and similarly $\pi \circ 1_{G} \times \mu=p_{G}$, so the "identity diagram" is commutative.

Finally, define $\alpha: G \rightarrow G$ by setting $\alpha(g)$, for $g \in \gamma^{-1}(a)$, equal to the inverse of $g$ in the group $\left(g^{-1}(a), \pi_{a}\right)$. If $g \in \gamma^{-1}(a)$, then $\alpha(g) \in \gamma^{-1}(a)$, so $\gamma \circ \alpha=\gamma$ and $\alpha \in$ $\operatorname{hom}((G, \gamma),(G, \gamma))$. Now

$$
\pi \circ \alpha \times 1_{G} \circ \Delta(g)=\pi \circ \alpha \times 1_{G}(g, g)=\pi(\alpha(g), g)=\pi_{a}(\alpha(g), g)=\mu(a)=\mu \circ \gamma(g),
$$

and so the "inverse diagram" is commutative. This completes the proof.

We now turn our attention to the structure of the endomorphism near-ring (End $((G, \gamma)),+, \circ)$ of an arbitrary group object $((G, \gamma), \pi, \mu, \alpha)$.

It is immediate that $f \in \operatorname{End}((G, \gamma))$ if and only if $f\left(\gamma^{-1}(a)\right) \subseteq \gamma^{-1}(a)$ for each $a \in A$. Hence $f=\cup_{a \in A} f_{a}$ where $f_{a}=f \mid \gamma^{-1}(a)$. For $f, h \in \operatorname{End}((G, \gamma)), f+h=\pi \circ[f, h]$, so $(f+h)(g)=\pi(f(g), h(g))=\pi_{a}\left(f_{a}(g), h_{a}(g)\right)=f_{a}(g)+h_{a}(g)$. This suggests 
Theorem 2. $\operatorname{End}((G, \gamma)) \cong \bigoplus \Sigma_{a \in A}^{*} \operatorname{Map}\left(\gamma^{-1}(a), \gamma^{-1}(a)\right)$

Proof. The discussion above shows that the map $f \rightarrow\left(f_{a}\right)_{a \in A}$ is a bijection. Let $+_{a}$ be defined on $\operatorname{Map}\left(\gamma^{-1}(a), \gamma^{-1}(a)\right)$ by $\left(f_{a}+{ }_{a} h_{a}\right)(g)=\pi_{a}\left(f_{a}(g), h_{a}(g)\right)$. Then one easily gets $f+h \rightarrow\left(f_{a}+{ }_{a} h_{a}\right)_{a \in A}=\left(f_{a}\right)_{a \in A}+\left(h_{a}\right)_{a \in A}$. Similarly, for $g \in \gamma^{-1}(a), \quad(f \circ h)(g)=$ $f[h(g)]=f_{a}\left(h_{a}(g)\right)=\left(f_{a} \circ h_{a}\right)(g)$, so $f \circ g \rightarrow\left(f_{a} \circ h_{a}\right)_{a \in A}$, and we have the isomorphism.

Similarly one gets

Theorem 3. $\operatorname{hom}((X, \eta),(G, \gamma)) \cong \bigoplus \Sigma^{*} \operatorname{Map}\left(\eta^{-1}(a), \gamma^{-1}(a)\right)$, and if $f \in \operatorname{End}((G, \gamma)), h \in \operatorname{hom}((X, \eta),(G, \gamma)), f \rightarrow\left(f_{a}\right)$, and $h \rightarrow\left(h_{a}\right)$,

then

$f \circ h \in \operatorname{hom}((X, \eta),(G, \gamma))$ and $f \circ h \rightarrow\left(f_{a} \circ h_{a}\right) \in \bigoplus \sum^{*} \operatorname{Map}\left(\eta^{-1}(a), \gamma^{-1}(a)\right)$

\section{The fibred product near-rings, etc. for $\mathscr{G}$, the category of groups}

Fix a group $G$. We'll first determine the group objects of $\mathscr{G}(G)$.

Suppose $((X, \eta), \pi, \mu, \alpha)$ is a group object of $\mathscr{G}(G)$. Let $A=\operatorname{ker} \eta$ and $i: A \rightarrow X$ be the insertion map. We must have $\mu$ as the "identity morphism," so $\eta \circ \mu=1_{G}$. This is exactly what is needed to say that

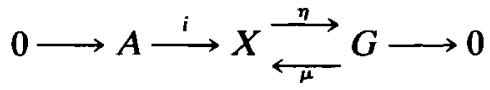

is split exact, thus $X$ is isomorphic to a semidirect product $A \times{ }_{\theta} G$ for some homomorphism $\theta: G \rightarrow$ Aut $A$, that $(a, x)+(b, y)=(a+\theta(x)(b), x+y)$ defines the operation in $A \times G$ for the group $A \times{ }_{\theta} G$, and that $\mu(g)=(0, g)$.

We shall now see that $A$ must be abelian. Consider the "identity diagram." The elements of $G \times{ }_{G} X$ are $(g, x)$ where $\eta x=g$. That is, the elements of $G \times{ }_{G} X$ are exactly the $(\eta x, x), x \in X$. Now $p_{X}(\eta x, x)=x$ and $\pi \circ \mu \times 1_{G}(\eta x, x)=\pi(\mu \eta x, x)$. Hence

$$
\pi(\mu \eta x, x)=x
$$

Similarly one gets

$$
\pi(x, \mu \eta x)=x .
$$

Recall $(a, b) \in X \times{ }_{G} X$ if and only if $\eta a=\eta b$. For such an $(a, b),(-\mu \eta b,-\mu \eta a)$, $(\mu \eta \boldsymbol{b}, \boldsymbol{b}) \in X \times{ }_{G} X$.

But

so

$$
(a, b)=(a, \mu \eta a)+(-\mu \eta b,-\mu \eta a)+(\mu \eta b, b)
$$

$$
\begin{aligned}
\pi(a, b) & =\pi(a, \mu \eta a)+\pi(-\mu \eta b,-\mu \eta a)+\pi(\mu \eta b, b) \\
& =a+\pi(\mu \eta \mu \eta(-b), \mu \eta(-b))+b \\
& =a+\mu \eta(-b)+b
\end{aligned}
$$

since $\eta a=\eta b$. 
Suppose $a, b \in A=\operatorname{ker} \eta$. Then

and

$$
\pi(a, b)=a+b
$$

$$
\begin{aligned}
\pi(a, b) & =\pi[(a, \mu \eta b)+(-\mu \eta b,-\mu \eta b)+(\mu \eta b, b)] \\
& =\pi[(a, 0)+(0,0)+(0, b)] . \\
& =\pi[(a, 0)+(0, b)]=\pi[(0, b)+(a, 0)] \\
& =\pi(0, b)+\pi(a, 0) \\
& =\pi(\mu \eta b, b)+\pi(a, \mu \eta a)=b+a .
\end{aligned}
$$

Hence $A$ is abelian.

We'll now see that the "inverse morphism" $\alpha$ is defined by

$$
\alpha(x)=\mu \eta(x)-x+\mu \eta(x) .
$$

Since $\operatorname{hom}\left((X, \eta), \quad\left(G, 1_{G}\right)\right)=\{\eta\}, t_{X}=\eta$. The commutativity of the "inverse diagram" yields

$$
\begin{aligned}
\mu \circ \eta(x) & =\pi \circ \alpha \times 1_{X} \circ\left[1_{X}, 1_{X}\right](x) \\
& =\pi \circ(\alpha x, x)=\alpha x+\mu \eta(-x)+x
\end{aligned}
$$

Hence

$$
\alpha(x)=\mu \eta(x)-x+\mu \eta(x) .
$$

We now have one half of

Theorem 4. The group objects of $\mathscr{G}(G)$ are exactly the quadruples

$$
\left(\left(A \times{ }_{\theta} G, \eta\right), \pi, \mu, \alpha\right)
$$

where $A$ is abelian, the short exact sequence

$$
0 \longrightarrow A \stackrel{i}{\longrightarrow} A \times{ }_{\theta} G \stackrel{\eta}{\stackrel{\mu}{\longrightarrow}} G \longrightarrow 0
$$

is split, where $i$ is the insertion map $i(a)=(a, 0)$, where $\eta \circ \mu=1_{G}$, where $\mu(g)=(0, g)$, where $\pi$ is defined by

$$
\pi(x, y)=x-\mu \eta(y)+y,
$$

and where $\alpha$ is defined by

$$
\alpha(x)=\mu \eta(x)-x+\mu \eta(x)
$$

Proof. To finish the proof of this theorem we need only show that split short exact sequences

$$
0 \longrightarrow A \stackrel{i}{\longrightarrow} A \times{ }_{\theta} G \stackrel{\eta}{\longleftrightarrow} G \longrightarrow 0
$$

where $A$ is abelian and $\mu(g)=(0, g)$, determine a group object $\left(\left(A \times{ }_{\theta} G, \eta\right), \pi, \mu, \alpha\right)$ of $\mathscr{G}(G)$. We have already that $\left(A \times{ }_{\theta} G, \eta\right)$ is an object of $\mathscr{G}(G)$ and that $\mu \in$ 
$\operatorname{hom}\left(\left(G, 1_{G}\right),\left(A \times{ }_{\theta} G, \eta\right)\right)$. For $\pi$ and $\alpha$ as defined in the theorem, we shall see that they are morphisms of $\mathscr{G}(G)$ and that the appropriate diagrams are commutative.

First, consider $\alpha$. If one lets $x=(a, g), y=(b, h) \in A \times{ }_{\theta} G$ and remembers that $\eta(a, g)=g$ and $\mu(g)=(0, g)$, one can see that $\alpha: A \times{ }_{\theta} G \rightarrow A \times{ }_{\theta} G$ is a group homomorphism. Since $\eta \alpha(x)=\eta \mu \eta(x)-\eta(x)+\eta \mu \eta(x)=\eta(x)$, it follows that $\alpha$ is a morphism of $\mathscr{G}(G)$.

For $\pi$, recall that $((a, g),(b, h)) \in\left(A \times{ }_{\theta} G\right) \times{ }_{G}\left(A \times{ }_{\theta} G\right)$ implies $\eta(a, g)=\eta(b, h)$, so $g=h$. With this in mind and with a fair amount of careful computation, one sees that $\pi$ is in fact a group homomorphism. To see that $\pi$ is a morphism of $\mathscr{G}(G)$, note that

so $\eta \circ \pi=\bar{\eta}$ as desired.

$$
\begin{aligned}
\eta \circ \pi((a, g),(b, h)) & =\eta[(a, g)-\mu \eta(b, h)+(b, h)] \\
& =\eta[(a, g)-(0, h)+(b, h)] \\
& =\eta[(a, g)+(\theta(-h)(b), 0)] \\
& =\eta(a+\theta(g) \theta(-h)(b), g)=g \\
& =\bar{\eta}((a, g),(b, h)),
\end{aligned}
$$

We now take up the matter of commutativity of the various group object diagrams. We'll first verify that $\pi$ has the "associative property." Note that

$$
\begin{aligned}
\pi \circ \pi \times 1(((a, g),(b, h)),(c, k)) & =\pi[\pi((a, g),(b, h)),(c, k)] \\
& =\pi[(a+\theta(g) \theta(-h)(b), g),(c, k)] \\
& =(a+\theta(g) \theta(-h)(b)+\theta(g) \theta(-k)(c), g) \\
& =(a+b+c, g), \text { since } g=h=k .
\end{aligned}
$$

Next note that

$$
\begin{aligned}
\pi \circ 1 \times \pi((a, g),((b, h),(c, k))) & =\pi((a, g), \pi((b, h),(c, k))) \\
& =\pi((a, g),(b+\theta(h) \theta(-k)(c), h) \\
& =(a+\theta(g) \theta(-h)(b+\theta(h) \theta(-k)(c)), g) \\
& =(a+b+c, g), \text { since } g=h=k .
\end{aligned}
$$

Hence, $\pi$ has the "associative property."

Next is $\mu$ and the "identity property." For $\left.(g,(a, g)) \in G \times{ }_{G}\left(A \times{ }_{\theta} G\right)\right)$, an arbitrary element,

$$
\begin{aligned}
\pi \circ \mu \times 1(g,(a, g)) & =\pi(\mu(g),(a, g)) \\
& =\pi((0, g),(a, g))=(0+\theta(g) \theta(-g)(a), g) \\
& =(a, g)=P_{A \times{ }^{g} G}(g,(a, g)),
\end{aligned}
$$

so

$$
\pi \circ \mu \times 1_{A \times{ }_{\theta} G}=p_{A \times{ }_{\theta} G}
$$

Similarly one gets

$$
\pi \circ 1_{A \times{ }_{\theta} G} \times \mu=p_{A \times{ }_{\theta} C}
$$

and so the "identity diagram" is commutative. 
Finally, we consider the "inverse diagram" and the morphism $\alpha$. For $(a, g) \in$ $A \times{ }_{\theta} G$,

$$
\begin{aligned}
\pi \circ \alpha \times 1_{A \times \theta} \circ \circ \Delta(a, g) & =\pi(\alpha(a, g),(a, g)) \\
& =\pi(\mu \eta(a, g)-(a, g)+\mu \eta(a, g),(a, g)) \\
& =\pi\left((0, g)+\left(\theta(g)^{-1}(-a),-g\right)+(0, g),(a, g)\right) \\
& =\pi((-a, 0)+(0, g),(a, g))=\pi((-a, g),(a, g)) \\
& =(-a, g)-\mu \eta(a, g)+(a, g) \\
& =(-a, g)+(0,-g)+(a, g)=(0, g)
\end{aligned}
$$

and

$$
\mu \circ t_{A x_{\theta} G}(a, g)=\mu(\eta(a, g))=(0, g) .
$$

Thus the "inverse diagram" is commutative and so this completes the proof.

We now turn our attention to determining the structure of the endomorphism near-rings $\left(\operatorname{End}\left(A \times{ }_{\theta} G, \eta\right),+, \circ\right)$ for a group object $\left(\left(A \times{ }_{\theta} G, \eta\right), \pi, \mu, \alpha\right)$ of $\mathscr{G}(G)$. For $f \in \operatorname{End}\left(A \times{ }_{\theta} G, \eta\right)$ we have $\eta \circ f=\eta$ and consequently

$$
g=\eta(a, g)=\eta \circ f(a, g)=\eta(\bar{a}, \bar{g})=\bar{g} \text {. }
$$

So we have

$$
f(a, g)=(\bar{a}, g) .
$$

Also, $\operatorname{ker} f \subseteq \operatorname{ker} \eta=\{(a, 0) \mid a \in A\}$. Suppose

$$
f(a, 0)=(l(a), 0) .
$$

Then one gets that $l \in \operatorname{Hom}(A, A)$ directly.

Suppose $f(0, g)=(b(g), g)$. Then

$$
f(a, g)=f[(a, 0)+(0, g)]=(l(a), 0)+(b(g), g)=(l(a)+b(g), g) .
$$

Also,

$$
\begin{aligned}
\left(b\left(g+g^{\prime}\right), g+g^{\prime}\right) & =f\left(0, g+g^{\prime}\right)=f(0, g)+f\left(0, g^{\prime}\right) \\
& =(b(g), g)+\left(b\left(g^{\prime}\right), g^{\prime}\right)=\left(b(g)+\theta(g) b\left(g^{\prime}\right), g+g^{\prime}\right) .
\end{aligned}
$$

So $b\left(g+g^{\prime}\right)=b(g)+\theta(g) b\left(g^{\prime}\right)$ and $b: G \rightarrow A$ is a crossed homorphism. From

$$
\begin{aligned}
f\left[(a, g)+\left(a^{\prime}, g^{\prime}\right)\right] & =f(a, g)+f\left(a^{\prime}, g^{\prime}\right) \\
& =(l(a)+b(g), g)+\left(l\left(a^{\prime}\right)+b\left(g^{\prime}\right), g^{\prime}\right) \\
& =\left(l(a)+b(g)+\theta(g)\left[l\left(a^{\prime}\right)+b\left(g^{\prime}\right)\right], g+g^{\prime}\right)
\end{aligned}
$$

and

$$
f\left(a+\theta(g)\left(a^{\prime}\right), g+g^{\prime}\right)=\left(l\left(a+\theta(g)\left(a^{\prime}\right)+b\left(g+g^{\prime}\right)\right), g+g^{\prime}\right)
$$

we get

$$
\theta(g) l\left(a^{\prime}\right)=l\left(\theta(g)\left(a^{\prime}\right)\right)
$$


and so

$$
\theta(g) \circ l=l \circ \theta(g) .
$$

Hence $l$ commutes with each $\theta(g) \in \theta(G) \subseteq \operatorname{Hom}(A, A)$.

Let $\mathscr{C}(A, G)=\{l \in \operatorname{Hom}(A, A) \mid l \circ \theta(g)=\theta(g) \circ l$ for all $g \in G\}$. Then $\mathscr{C}(A, G)$ is a ring. If $Z_{\theta}^{\prime}(G, A)$ denotes all crossed homomorphisms from $G$ to $A$ with respect to $\theta$, then $Z_{\theta}(G, A)$ is a unitary $\mathscr{C}(A, G)$-module.

One easily gets a bijection between $\operatorname{End}\left(A \times{ }_{\theta} G, \eta\right)$ and $\mathscr{C}(A, G) \times Z_{\theta}^{\prime}(G, A)$. Let $f, f^{\prime} \in \operatorname{End}\left(A \times{ }_{\theta} G, \eta\right)$ correspond to $(l, b),\left(l^{\prime}, b^{\prime}\right) \in \mathscr{C}(A, G) \times Z_{\theta}^{\prime}(G, A)$, respectively. Now $f+f^{\prime}=\pi \circ\left[f, f^{\prime}\right]$, so

$$
\begin{aligned}
\left(f+f^{\prime}\right)(a, g) & =\pi \circ\left[f, f^{\prime}\right](a, g)=\pi\left[f(a, g), f^{\prime}(a, g)\right] \\
& =f(a, g)-\mu \eta f^{\prime}(a, g)+f^{\prime}(a, g) \\
& =(l(a)+b(g), g)-\mu \eta\left(l^{\prime}(a)+b^{\prime}(g), g\right)+\left(l^{\prime}(a)+b^{\prime}(g), g\right) \\
& =(l(a)+b(g), 0)+\left(l^{\prime}(a)+b^{\prime}(g), g\right) \\
& =\left(\left(l+l^{\prime}\right)(a)+\left(b+b^{\prime}\right)(g), g\right) .
\end{aligned}
$$

Hence $f+f^{\prime}$ corresponds to $\left(l+l^{\prime}, b+b^{\prime}\right)$. Similarly, $f \circ f^{\prime}(a, g)=f\left(l^{\prime}(a)+b^{\prime}(g), g\right)$

$$
=\left(l \circ l^{\prime}(a)+\left(l \circ b^{\prime}+b\right)(g), g\right) \text {, }
$$

and so $f \circ f^{\prime}$ corresponds to $\left(l \circ l^{\prime}, l \circ b^{\prime}+b\right)$. We have therefore Theorem 5 . The map

Theorem 5. The map

$$
\begin{gathered}
F: \operatorname{End}\left(A \times{ }_{\theta} G, \eta\right) \rightarrow \mathscr{C}(A, G) \times Z_{\theta}^{\prime}(G, A) \text { defined by } \\
F(f)=(l, b)
\end{gathered}
$$

where $f(a, g)=(l(a)+b(g), g)$, is a near-ring isomorphism onto the abstract affine near-ring $\left(\mathscr{C}(A, G) \times Z_{\theta}^{\prime}(G, A),+, \cdot\right)$.

We now determine the structure of the $\operatorname{End}\left(A \times{ }_{\theta} G, \eta\right)$-groups $\operatorname{hom}((X, \epsilon)$, $\left(A \times{ }_{\theta} G, \eta\right)$ ) for objects $(X, \epsilon)$ in $\mathscr{G}(G)$, where $X$ is an extension of an abelian $B$ by $G$ realizing $\lambda$. We may suppose that $X$ has factor set $f: G \times G \rightarrow B, X=B \times{ }_{\lambda}^{f} G$,

$$
(a, g)+\left(a^{\prime}, g^{\prime}\right)=\left(a+\lambda(g)\left(a^{\prime}\right)+f\left(g, g^{\prime}\right), g+g^{\prime}\right),
$$

and $\epsilon(a, g)=g$. Consider $F \in \operatorname{hom}\left((X, \epsilon),\left(A \times{ }_{\theta} G, \eta\right)\right)$. Since $\eta \circ F=\epsilon$, we have $F(a, g)=(\bar{a}, g)$. From $F(a, 0)=(l(a), 0)$ we get $l \in \operatorname{Hom}(B, A)$, and from $F(0, g)=$ $(b(g), g)$, we get $F(a, g)=(l(a)+b(g), g)$.

Now

$$
\begin{aligned}
F\left[(0, g)+\left(0, g^{\prime}\right)\right] & =F\left(f\left(g, g^{\prime}\right), g+g^{\prime}\right) \\
& =\left(l \circ f\left(g, g^{\prime}\right)+b\left(g+g^{\prime}\right), g+g^{\prime}\right)
\end{aligned}
$$

and

Consequently

$$
\begin{aligned}
F(0, g)+F\left(0, g^{\prime}\right) & =(b(g), g)+\left(b\left(g^{\prime}\right), g^{\prime}\right) \\
& =\left(b(g)+\theta(g) b\left(g^{\prime}\right), g+g^{\prime}\right) .
\end{aligned}
$$

$$
b\left(g+g^{\prime}\right)=b(g)+\theta(g) b\left(g^{\prime}\right)-l \circ f\left(g, g^{\prime}\right)
$$


We conclude that $l \circ f \in B_{\theta}^{2}(G, A)$, the coboundaries of $G$ by $A$. Similar to the case where $X=A \times{ }_{\theta} G$, we see that $l \in \mathscr{C}(\lambda, \theta)$ where

$$
\mathscr{C}(\lambda, \theta)=\{l \in \operatorname{Hom}(B, A) \mid \theta(g) \circ l=l \circ \lambda(g) \text { for all } g \in G\},
$$

a subgroup of $\operatorname{Hom}(B, A)$. The condition (*) implies that $l$ belongs to the subgroup

$$
A(f)=\left\{l \in \mathscr{C}(\lambda, \theta) \mid l \circ f \in B_{\theta}^{2}(G, A)\right\} .
$$

For $l \in A(f)$, define

$$
\mathscr{B}(l \circ f)=\left\{b: G \rightarrow A \mid b\left(g+g^{\prime}\right)=b(g)+\theta(g) b\left(g^{\prime}\right)-l \circ f\left(g, g^{\prime}\right)\right\}
$$

and

$$
\overline{\mathscr{B}}(f)=\bigcup_{l \in A(f)} \mathscr{B}(l \circ f)
$$

We have

Lemma 6. $\overline{\mathscr{B}}(f)$ is an abelian group, and

$$
\mathscr{B}(0)=Z_{\theta}^{\prime}(G, A)
$$

is a subgroup.

Proof. Since $\operatorname{Map}(G, A)$ is an abelian group, one needs only to show that $b_{1}-b_{2} \in \overline{\mathscr{B}}(f)$ for arbitrary $b_{1}, b_{2} \in \overline{\mathscr{B}}(f)$. This follows immediately from the fact that $A(f)$ is a subgroup of $\mathscr{C}(\lambda, \theta)$. Obviously $\mathscr{B}(0)=Z_{\theta}^{\prime}(G, A)$ and is a subgroup.

Lemma 7. For $b \in \mathscr{B}(l \circ f), \mathscr{B}(l \circ f)=\mathscr{B}(0)+b$.

Proof. For $b_{1} \in \mathscr{B}(0)$, it is direct to show that $b_{1}+b \in \mathscr{B}(l \circ f)$, so $\mathscr{B}(0)+b \subseteq$ $\mathscr{B}(l \circ f)$. Likewise, if $b_{2} \in \mathscr{B}(l \circ f)$, it follows that $b_{2}-b \in \mathscr{B}(0)$, so $b_{2}=c+b$ for some $c \in \mathscr{B}(\mathbf{0})$. Hence $\mathscr{B}(l \circ f) \subseteq \mathscr{B}(0)+b$.

Lemma 8. $\mathscr{B}\left(\left(l_{1}+l_{2}\right) \circ f\right)=\mathscr{B}\left(l_{1} \circ f\right)+\mathscr{B}\left(l_{2} \circ f\right)$

The proof is direct.

Let $n: \overline{\mathscr{B}}(f) \rightarrow \overline{\bar{B}}(f) / \mathscr{B}(0)$ be the natural map, and define $h: A(f) \rightarrow \overline{\mathscr{B}}(f) / \mathscr{B}(0)$ by $h(l)=\mathscr{B}(l \circ f)$. Then $(\overline{\mathscr{B}}(f), n)$ and $(A(f), h)$ are objects in $\mathscr{G}(\overline{\mathscr{B}}(f) / \mathscr{B}(0))$, and we have the following

Theorem 9. As a group,

$$
\operatorname{hom}\left(\left(B \times{ }_{\lambda}^{f} G, \epsilon\right),\left(A \times{ }_{\theta} G, \eta\right)\right)
$$

is isomorphic to the fibred product

$$
(A / f), h) \times_{\overline{\mathscr{A}}(f) / \Re(0)}(\overline{\mathscr{B}}(f), n)
$$

and if $F \in \operatorname{End}\left(A \times{ }_{\theta} G, \eta\right)$ corresponds to $(l, b)$ as in Theorem 5, and $F^{\prime} \in$ hom $\left(\left(B \times\{G, \epsilon),\left(A \times{ }_{\theta} G, \eta\right)\right)\right.$ corresponds to $\left(l^{\prime}, b^{\prime}\right)$ as above, then $F \circ F^{\prime}$ corresponds 
to $\left(l \circ l^{\prime}, l \circ b^{\prime}+b\right)$, which is, of course, analogous to the multiplication for an abstract affine near-ring.

Proof. We already have $F$ corresponding to

$$
(l, b) \in A(f) \times_{\overline{\mathscr{B}}(f) / \mathscr{B}(0)} \overline{\mathscr{B}}(f) .
$$

The rest is direct using the above lemmas.

The author wishes to express his appreciation to Prof. A. Fröhlich for stimulating conversations concerning the subject matter of this paper. This paper is actually built upon an example given in a seminar by $A$. Fröhlich and the basic idea of near-rings from group objects can be seen in Fröhlich's early papers on near-rings.

\section{REFERENCES}

(1) B. ECKMANN and P. J. HiLton, Group-like structures in general categories I: Multiplications and comultiplications, Math. Ann. 145 (1962), 227-255.

(2) B. ECKMANN and P. J. HiLton, Group-like structures in general categories II: Equalizers, limits, lengths, Math. Ann. 151 (1963), 150-186.

(3) B. EckmANn and P. J. Hilton, Group-like structures in general categories III: Primitive categories, Math Ann. 150 (1963), 165-187.

(4) A. FRÖHLICH, Distributively generated near-rings I. Ideal theory, Proc. London Math. Soc. 8 (1958), 76-94.

(5) H. GonSHOR, On abstract affine near-rings, Pacific J. Math. 14 (1964), 1237-1240.

(6) M. HolcomBE, Categorical representations of endomorphism near-rings, J. London Math. Soc. (2) 16 (1977), 21-37.

(7) S. MACLANE, Homology (Springer-Verlag, Berlin, 1963).

(8) S. MACLANE and G. Birkoff, Algebra (Macmillan, New York, 1967).

(9) J. RotMAN, The theory of groups: an introduction (Allyn and Bacon, Boston, 1965). 